\title{
Lung scanning in carcinoma of the bronchus
}

\author{
R. H. SECKER WALKER, J. L. PROVAN ${ }^{1}, J$. A. JACKSON, \\ and J. GOODW IN
}

The Medical Unit, University College Hospital Medical School, University Street, London W.C.1

\begin{abstract}
The lung scans of 101 patients with carcinoma of the bronchus have been compared with the bronchoscopic and radiographic findings. As the tumour approaches the hilum as judged by bronchoscopy or the chest radiograph the relative perfusion of the affected lung decreases. When the relative perfusion of the affected lung is less than one third of the total the tumour is likely to be inoperable. The reduction in perfusion is related to involvement of the vessels in the hilum by tumour and to a lesser extent to bronchial obstruction.

Defects in perfusion in the unaffected lung were seen in 53 patients and were usually due to chronic bronchitis and emphysema or inactive pulmonary tuberculosis.

In the management of patients with carcinoma of the bronchus lung scans are of value in predicting when a tumour is likely to be inoperable and also in giving an indication as to whether a pneumonectomy or lobectomy will be possible. In addition, defects in the contralateral lung may be sufficiently large to contraindicate surgery.
\end{abstract}

Lung scanning is a safe and effective way of demonstrating the relative pulmonary arterial blood flow to each lung (Sabiston and Wagner, 1964 ; Tauxe, Burchell, and Black, 1967) and has been used in this way to assess lung function in patients with carcinoma of the bronchus (Hatch, Maxfield, and Ochsner, 1965; Garnett, Goddard, Fraser, and Macleod, 1968). Defects in perfusion vary in size from those corresponding to the lesion visible on the chest radiograph to absent perfusion of an entire lung, while minor abnormalities are commonly seen in the opposite lung.

This paper compares the lung scan findings with the bronchoscopic and radiographic appearances in patients with carcinoma of the bronchus seen between 1967 and 1969, and discusses the practical value of this technique.

\section{PATIENTS AND METHODS}

The patients studied all had untreated carcinoma of the bronchus at the time of their lung scan.

The diagnosis of carcinoma of the bronchus was established by sputum cytology, biopsy at bronchoscopy or thoracotomy or from the histology of the resected tumour or at necropsy.

Each patient was given $1.5-2.0 \mathrm{mCi}$ of technetium $99 \mathrm{~m}$ labelled macroaggregated human serum albumin intravenously and the lung scans were made with a Picker Magnascanner III. Anterior and posterior colour scans and photoscans were produced.

1 Present address: Department of Surgery, The Wellesley Hospital, 160 Wellesley Street East, Toronto 5, Ontario, Canada
The relative pulmonary arterial blood flow (relative perfusion) of each lung has been determined from the colour dot scans by expressing the counts of radioactivity in the affected lung as a percentage of the total counts in the scan. This method correlates well with differential bronchospirometry as a measure of relative pulmonary blood flow (Chernick, LopezMajano, Wagner and Dutton, 1965 ; Rogers, Kuhl, Hyde, and Mayock, 1967; Garnett, Goddard, Machell, and Macleod, 1969).

In addition a semiquantitative method, also based on the colour dot scans, has been used to assess the size of the defects in perfusion in the unaffected lung. The recognition of such defects and the assessment of their size depend on a subjective comparison with the normal pattern of perfusion so that a truly quantitative method is not possible at present. A simple and rapid scoring method has been used in which each lung is divided into three zones on the anterior and posterior scans, and then each zone is scored from 0 to 4 (absent perfusion scoring 0 , and a normal pattern 4) depending on the size of the defect and the level of activity present. A normal lung would score $24(100 \%)$ and the defects have been expressed as a percentage of this. When the same observer scored the scans on a second occasion the maximum error was $7 \%$. By expressing the score for each lung as a percentage of the total score another estimate of relative perfusion can be made. There is an excellent correlation between this method of estimating relative perfusion and that derived from the counts of radio-activity in each lung $(r=0.986$, $\mathbf{P}<0.001$ ) (Secker Walker, Jackson, and Goodwin, 1970). 


\section{RESULTS}

Lung scans have been carried out on 101 patients with carcinoma of the bronchus. Fifty-two patients (44 men and 8 women) were considered on either bronchoscopic grounds (11) or because of poor respiratory function $\left(\mathrm{FEV}_{1}<1.5\right.$ litres) (16) or other diseases (5) or because of the presence of extrathoracic metastases (16) or superior vena caval obstruction (4) to be unsuitable for surgery. Forty-nine patients ( 35 men and 14 women) were considered on clinical and bronchoscopic grounds to have operable tumours, but three refused surgery so that 46 underwent thoracotomy. The average age of these 46 patients was $55 \cdot 2$, range 41-68 years, and the average age of the remaining 55 patients was $61 \cdot 7$, range $44-77$ years.

There were 21 squamous carcinomas, 16 oatcell or anaplastic carcinomas, and 9 adenocarcinomas in the patients undergoing surgery, and 27 squamous carcinomas, 13 oat-cell or anaplastic carcinomas, 5 adenocarcinomas, and 10 carcinomas of undetermined cell type in the other patients.

The relative perfusion of the affected lung, determined from the lung scans, has been com- pared with both the bronchoscopic and chest radiograph findings (Tables I and II). Thirty-four patients had normal bronchoscopies. The mean

T A B L E I

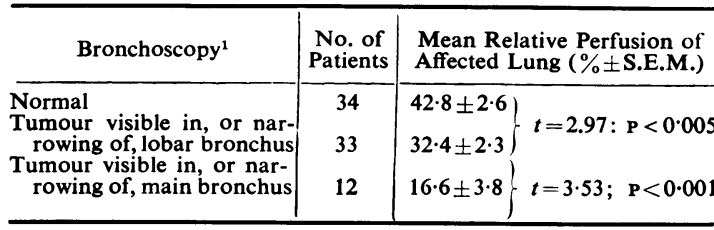

${ }^{1}$ Two patients with a widened main carina, two patients with stiff main bronchus, and 18 patients who did not have a bronchoscop are not included in this table.

\section{T A B L E I I}

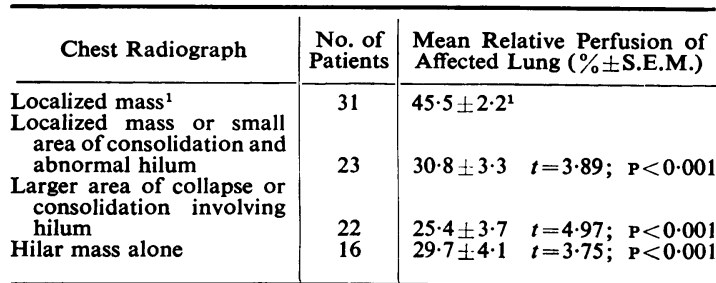

Five patients with normal chest radiographs and four with othe findings described in the text are not included.

${ }^{1}$ For Student's $t$ test the patients with a localized mass have been compared with each of the other groups.

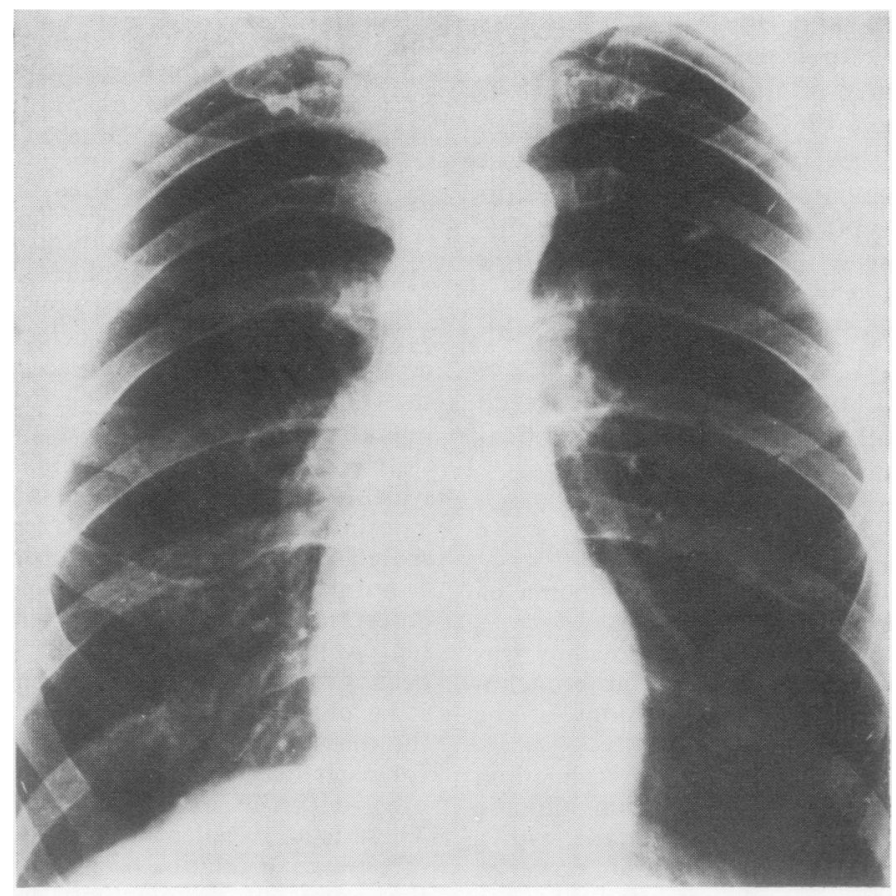

(a)

Legend to Fig. 1 a on page 26. 


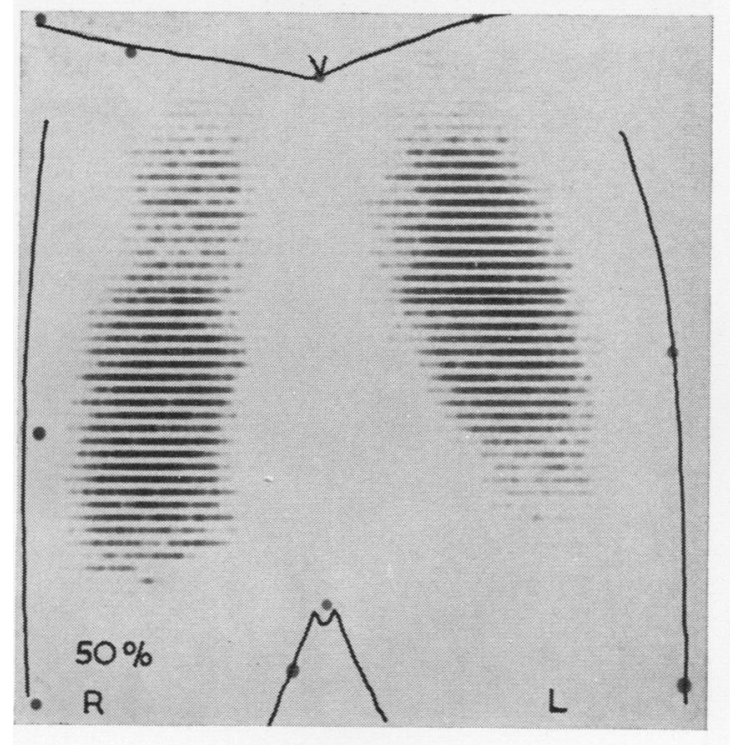

(b)

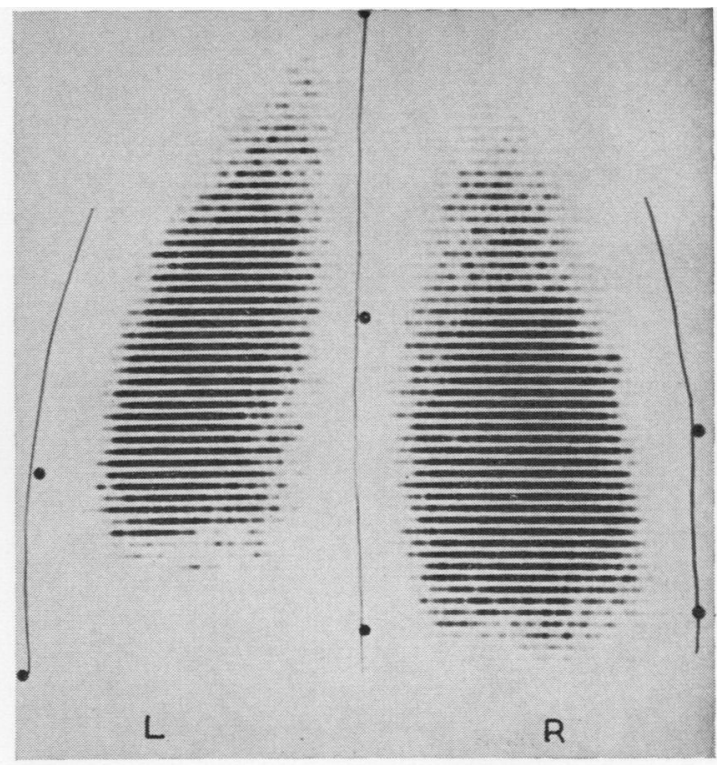

(c)

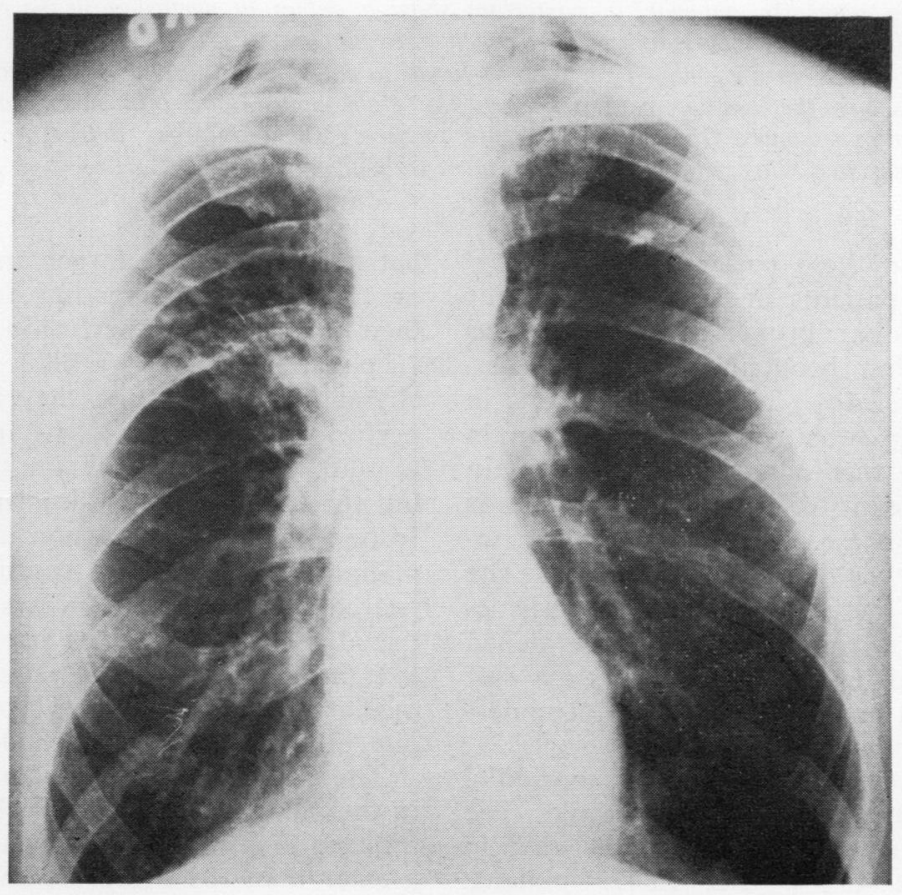

(d)

Legend to Fig. 1 b, c, d on page 26. 


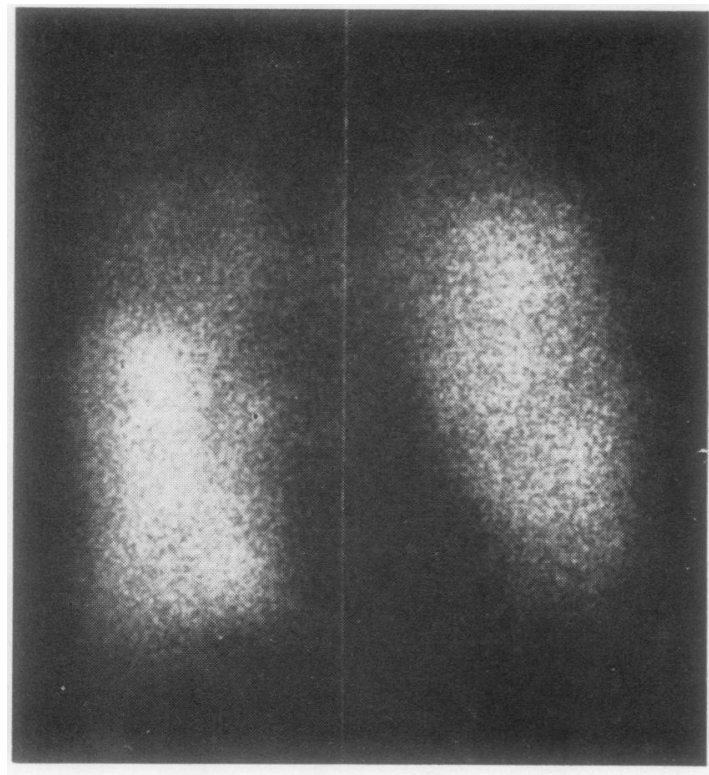

(e)

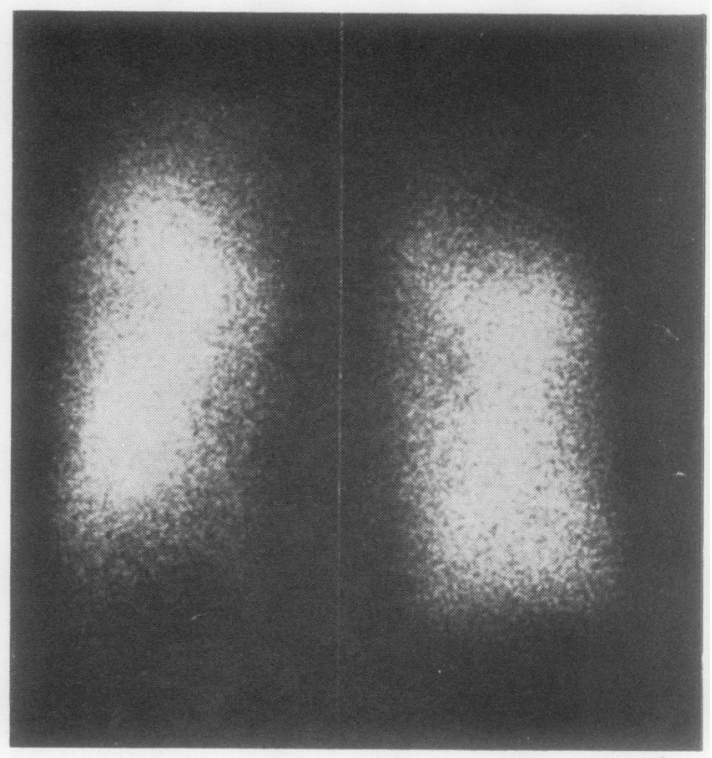

(f)

FIG. 1. Mr. J. E. aged 59. Squamous-cell carcinoma. Characteristic squamous carcinoma cells were found in the sputum in September 1967. Bronchoscopy and chest radiography (a), including tomography of the hilar regions, were normal. The lung scans (b, anterior, $\mathrm{c}$, posterior) show a defect in perfusion in the right upper lobe. Minor defects are present elsewhere. These findings were unchanged nine months later.

After a pneumonic illness in February 1970 he was found to have some patchy consolidation in the right upper lobe (d). At bronchoscopy the tumour was visible in the right upper lobe bronchus. Lung scans (e, anterior; $\mathrm{f}$, posterior) on the gamma camera (these are composite pictures) show a larger defect in the right upper lobe, but elsewhere the appearance is as before. He underwent a right upper lobectomy.

relative perfusion in these patients was $42 \cdot 8 \% \pm$ 2.6 (S.E.M.). In 33 patients in whom the tumour was visible in a lobar bronchus, or there was narrowing of a lobar bronchus, the mean relative perfusion was $32 \cdot 4 \% \pm 2 \cdot 3$. In 12 patients in whom the tumour was visible in the main bronchus or there was narrowing of the main bronchus, the mean relative perfusion was $16.6 \% \pm 3.8$ (one of these, in spite of the bronchoscopic appearance, was considered operable). The differences between these three groups are statistically significant. The main carina was widened in two patients and the main bronchus was stiff in two patients, but bronchoscopy was not undertaken in the remainder.

In 31 patients the chest radiograph showed a localized mass and the hilum was normal. The mean relative perfusion of the affected lung in these patients was $45 \cdot 5 \% \pm 2 \cdot 2$. In 23 patients there was a localized mass or small area of consolidation and the hilum was also abnormal : their mean relative perfusion was $30 \cdot 8 \% \pm 3 \cdot 3$. In 22 patients there was a larger area of either collapse or consolidation, spreading from the hilum, and their mean relative perfusion was $25.4 \% \pm 3.7$. In 16 patients there was a hilar mass without parenchymal shadowing and they had a mean relative perfusion of $29.7 \% \pm 4 \cdot 1$. In five patients the tumour was not visible on the chest radiograph but the lung scan was abnormal in each of them. In one of these, with a normal bronchoscopy and malignant cells in the sputum, the tumour appeared two and a half years later at the site of the abnormality and has been successfully resected (see Fig. 1). Two patients had a widened mediastinum, one bilateral pleural effusions and one bilateral peripheral shadows.

The mean relative perfusion of the affected lung in the patients with normal chest radiographs and those with a localized mass only are significantly greater than the mean relative perfusion of each of the other groups.

Figures 2 to 4 show examples of the lung scan findings. 


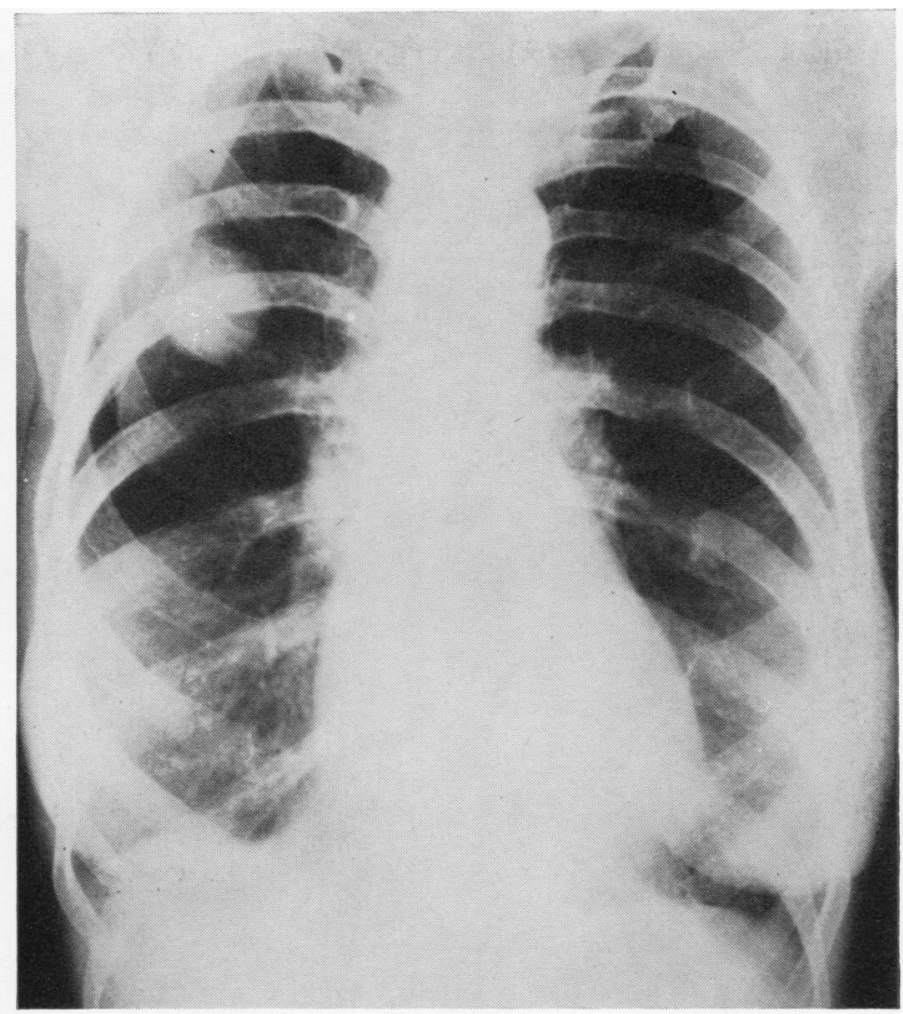

(a)

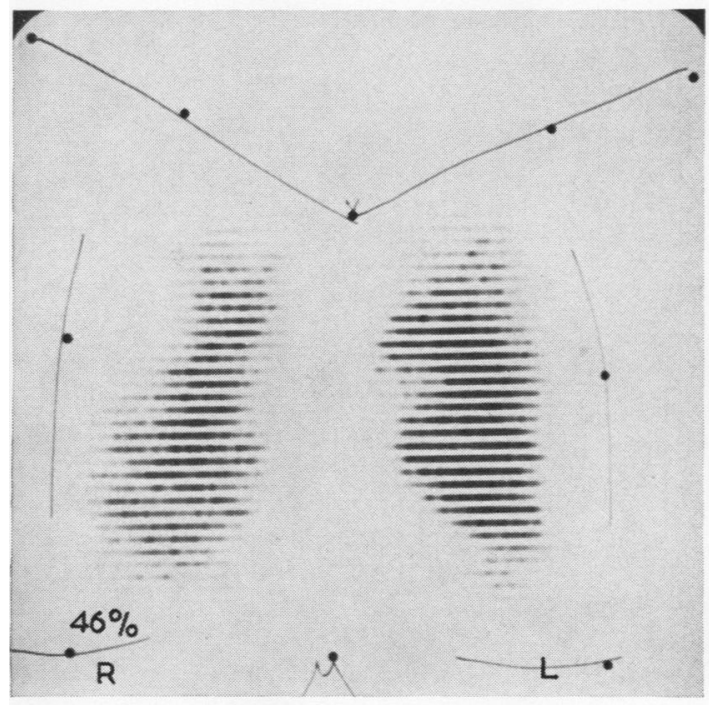

(b)

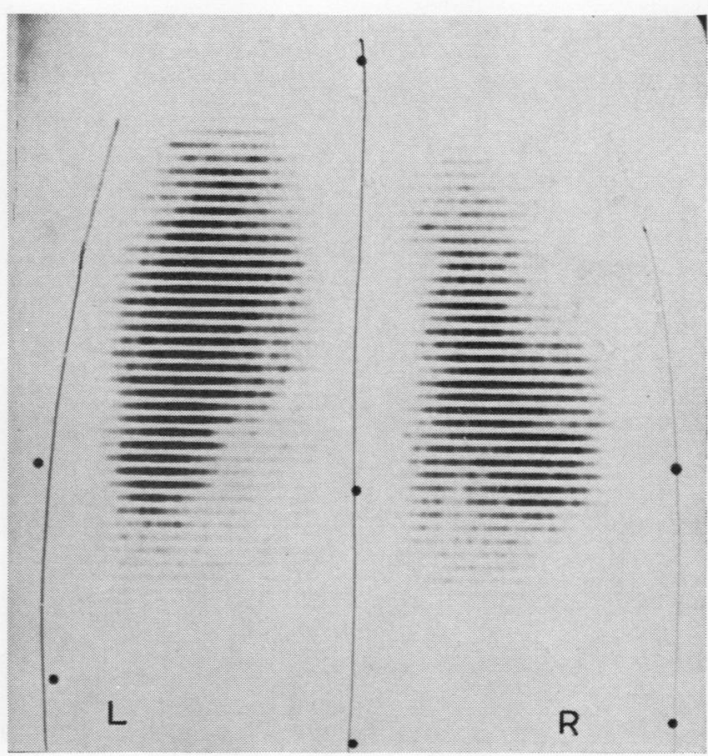

(c)

FIG. 2. Mrs. D. H. aged 59. Squamous-cell carcinoma. Bronchoscopy was normal. The lung scan shows a defect corresponding to the mass on the chest radiograph and several other defects in perfusion in each lung. The relative perfusion of the right lung was $46 \%$ and she underwent a right upper lobectomy. (b) Anterior scan; (c) posterior scan. 


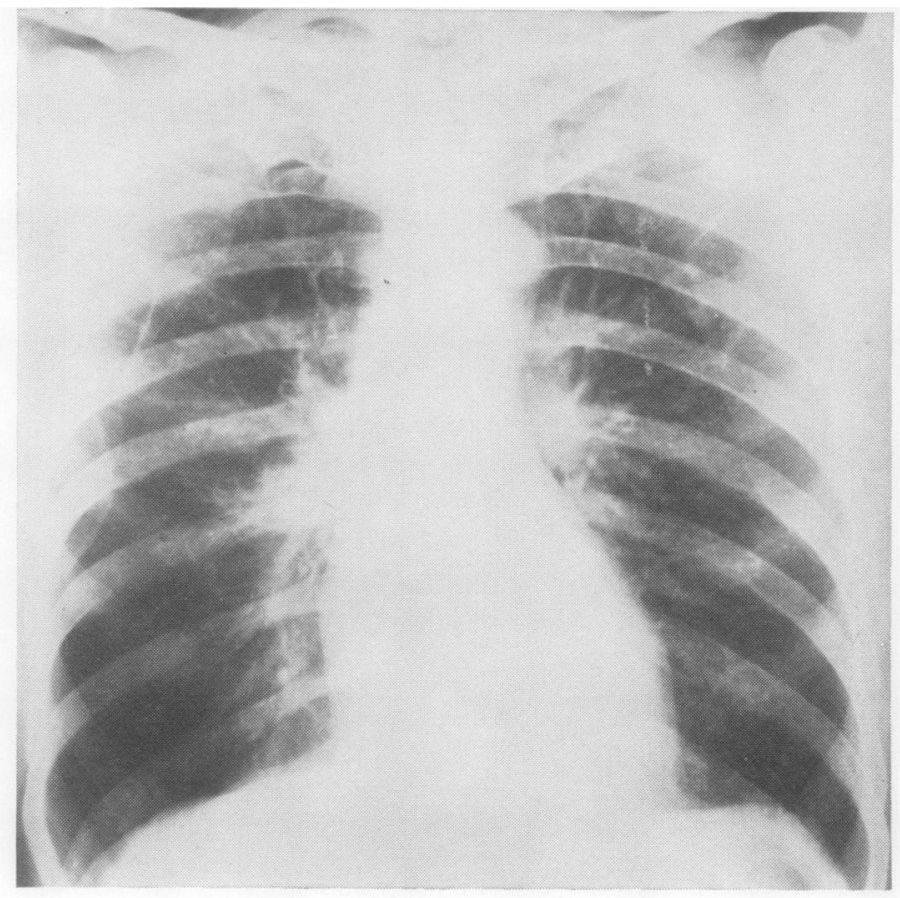

(a)

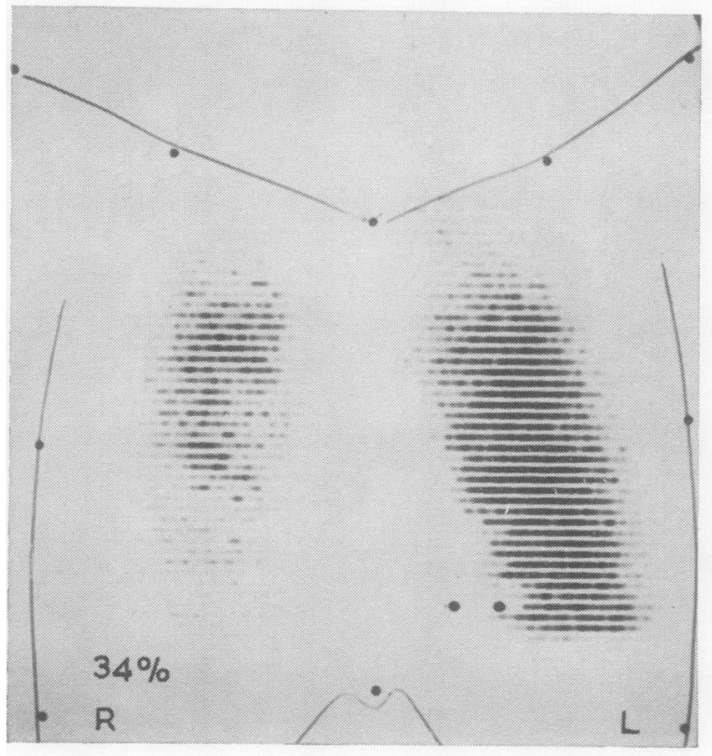

(b)

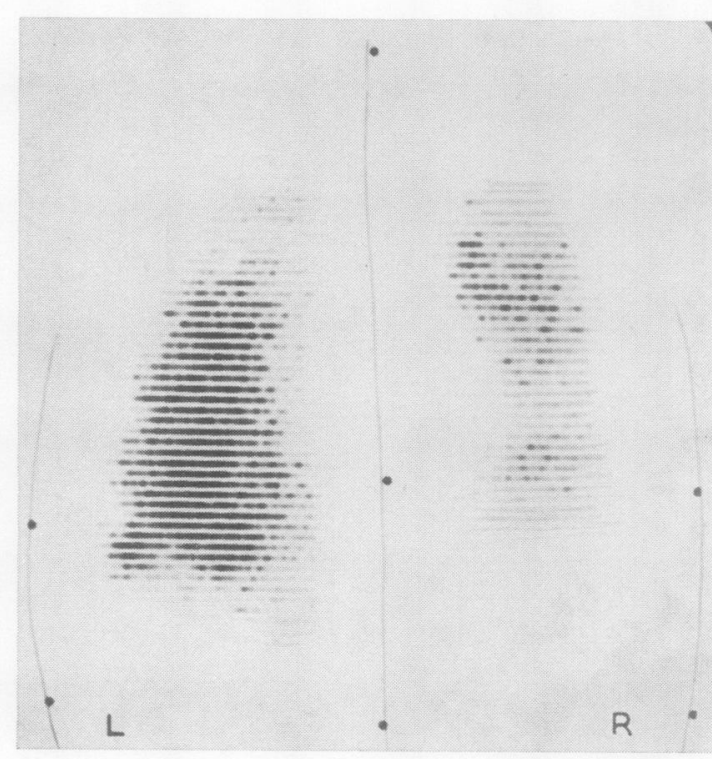

(c)

FIG. 3. Mr. J. I. aged 59. Squamous-cell carcinoma. At bronchoscopy the tumour was seen in the right lower lobe bronchus which was narrowed. The lung scan shows reduced perfusion of the right lung, especially in the lower two-thirds. The relative perfusion of the right lung was $34 \%$ and he underwent a right pneumonectomy. (b) Anterior scan; (c) posterior scan. 


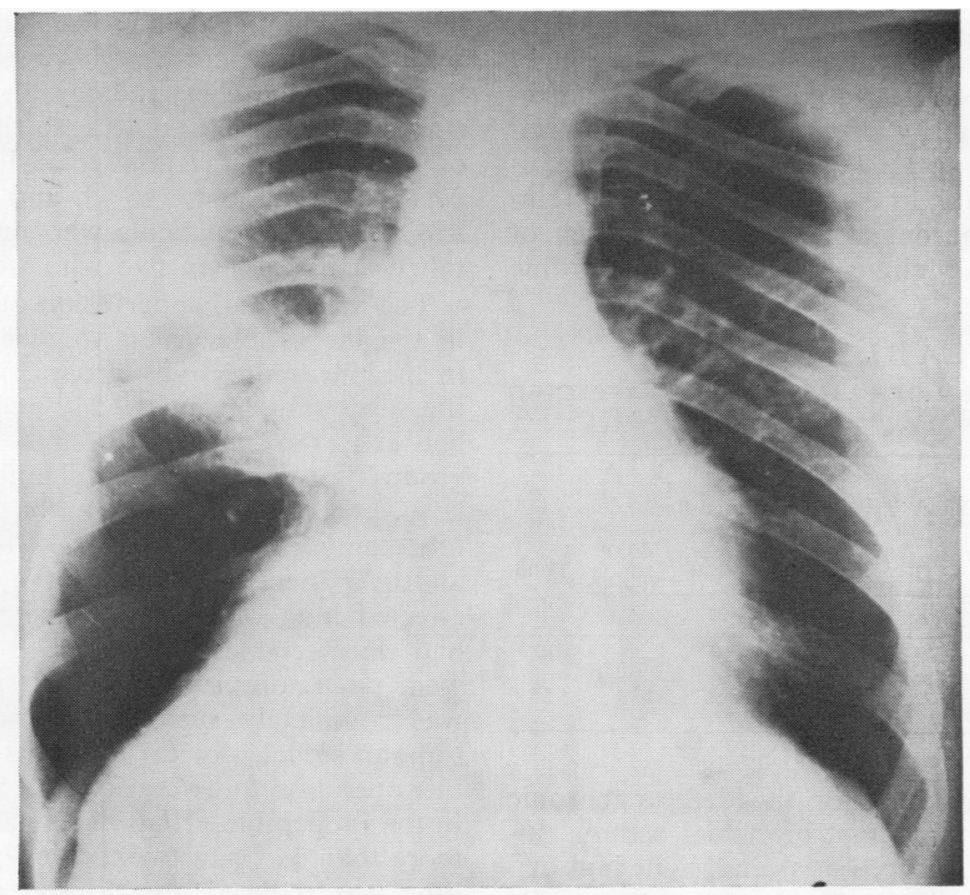

(a)

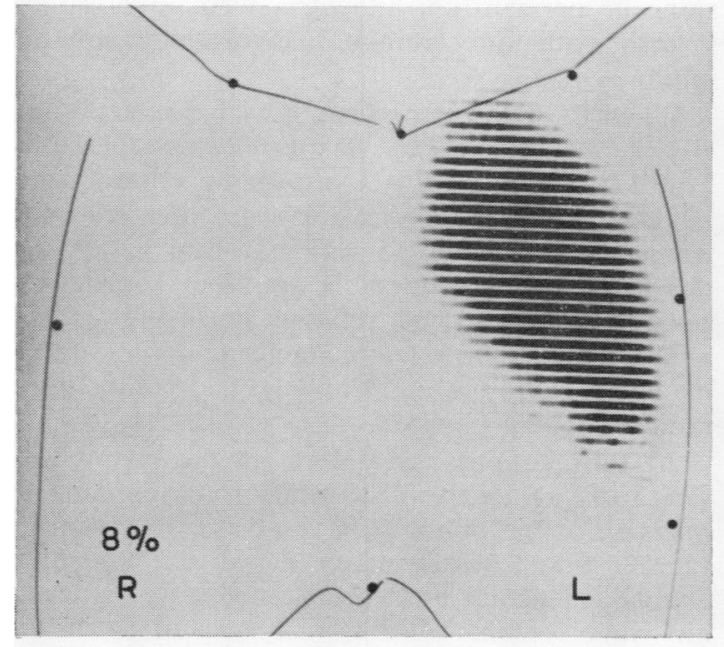

(b)

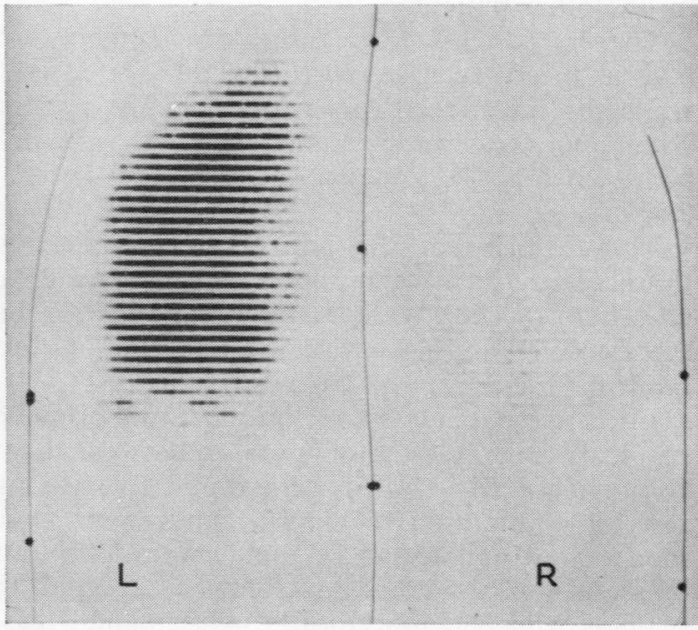

(c)

FIG. 4. Mr. J. G. aged 51. Oat-cell carcinoma. Bronchoscopy was normal. The lung scan shows very little perfusion of the right lung, the relative perfusion of this side being $8 \%$. He was treated with cyclophosphamide and died one month later from cerebral metastases. (b) Anterior scan; (c) posterior scan. 
Defects in the pattern of perfusion in the unaffected lung were seen in 53 patients (Table III), and such defects may give rise to misleadingly high values for the relative perfusion of the affected lung. The majority of such defects were relatively small, scoring less than $25 \%$ by the semiquantitative method, in 36 patients. In the remaining 17 patients the defects were larger. Seven of these patients had clinical evidence of chronic

\section{T A B L E I I I}

DEFECTS IN PATTERN OF PERFUSION IN UNAFFECTED LUNG AS DETERMINED BY THE SEMIQUANTITATIVE.

\begin{tabular}{l|ccc|c}
\hline & \multicolumn{3}{|c|}{$\begin{array}{c}\text { Findings in } \\
\text { Unaffected Lung }\end{array}$} & \multirow{2}{*}{} \\
\cline { 2 - 4 } & $\begin{array}{c}\text { Normal } \\
\text { Pattern }\end{array}$ & $\begin{array}{c}\text { Minor } \\
\text { Defects }\end{array}$ & $\begin{array}{c}\text { Major } \\
\text { Defects }\end{array}$ & Total \\
\hline Score & $<10 \%$ & $10-25 \%$ & $225 \%$ & \\
\hline $\begin{array}{l}\text { Patients undergoing thoracotomy } \\
\text { Patients not undergoing thora- } \\
\text { cotomy }\end{array}$ & 23 & 19 & 4 & 46 \\
\hline
\end{tabular}

lung disease to account for the defects (chronic bronchitis and emphysema, bronchial asthma, inactive pulmonary tuberculosis) and eight had evidence of metastatic spread either to the opposite lung (4) or outside the chest (4). The defects in perfusion in one patient were related to left ventricular failure associated with hypertension and ischaemic heart disease, and were not explained in one other patient.

The 16 patients with extrathoracic metastases had a mean relative perfusion of $28 \cdot 5 \% \pm 4 \cdot 6$, while the mean relative perfusion in the patients with poor respiratory function (16), ischaemic heart disease (4), and uraemia (1) was $41 \cdot 1 \% \pm$ $3 \cdot 4$.

The majority of the patients who underwent thoracotomy have been considered in more detail elsewhere (Secker Walker and Provan, 1969), but the essential findings, with the addition of three more patients, were as follows.

Forty-six patients had clinically and bronchoscopically operable tumours and underwent thoracotomy, but $16(35.0 \%)$ were found to have unresectable tumours, $15(32.5 \%)$ underwent pneumonectomy, and $15(32.5 \%)$ underwent lobectomy or segmental resection. As might be expected, the patients who had lobectomies or segmental resections had more normal bronchoscopies, less hilar involvement on the chest radiograph, and fewer abnormalities on their lung scans than the other two groups in whom the findings were similar. No abnormality was seen in the lung scans of two patients with small peripheral tumours. $\operatorname{In} \frac{\text { की }}{0}$ the remaining patients the defects ranged in $\frac{\mathrm{C}}{5}$ size from those corresponding to the size of the $\overline{\bar{c}}$ tumour on the chest radiograph to absent perfu- $\vec{\Phi}$ sion of the entire lung. Irregularities in the pattern $\frac{0}{0}$ of perfusion in the unaffected lung were seen in क 21 of these patients $(46 \%)$, and were larger than $\overrightarrow{0}$ $25 \%$ in the two patients who underwent segmental resection, and in two who were unresectable. $\vec{\omega}$

The mean relative perfusion of the affected lung $\stackrel{2}{\rightleftharpoons}$ in the inoperable group was $28.8 \% \pm 3 \cdot 8$ (S.E.M.), $\times$ in the pneumonectomy group $37 \cdot 7 \% \pm 2 \cdot 3$, and in $\%$ the lobectomy group $50 \cdot 2 \% \pm 1 \cdot 5$. The difference between the inoperable and pneumonectomy ${ }_{\omega}$ groups just fails to reach significance at the $5 \%$ 으 level, but both are significantly different from the lobectomy group, $\mathbf{P}<0.001$. With one exception all the patients with a relative perfusion of the affected lung of less than one third of the total had unresectable tumours. The exception under-went pneumonectomy. His left pulmonary artery $\vec{\theta}$ was completely surrounded and compressed by. tumour sufficiently far from its origin to enable this operation to be carried out. Six of the patients? in the inoperable group had relative perfusions of $\bar{\partial}$ more than $34 \%$. In two, perfusion of the opposite lung was reduced, due to spread of the tumour $\stackrel{2}{\square}$ across the mediastinum in one, and possibly also $\Rightarrow$ in the other, so that the relative perfusion of the $\frac{\text { o }}{3}$ affected lung was little reduced. In the other four $\frac{3}{5}$ the tumour was inoperable because the thoracic spine or pericardium and atria were involved by growth with only minimal involvement of the hilum.

When the relative perfusion lay between 34 and ${ }_{0}^{x}$ $40 \%$ a pneumonectomy was usually possible, only 3 . 2 of 12 patients in this group being unresectable. Above $40 \%$ a lobectomy or segmental resection was usually possible (15 patients), four patients in this group being inoperable and four requiring a pneumonectomy. The relative perfusion of theo affected lung in the two patients undergoing segmental resection was 59 and $60 \%$, making moreos radical surgery unwise.

At thoracotomy the defects in perfusion weren seen to be related to distortion or compression or invasion of the pulmonary vessels at the hilum by tumour tissue or secondary deposits in the hilar? glands. As would be expected, there was scarcelyes any involvement of the hilar structures in the patients undergoing lobectomy or segmental resec tion. Most involvement was seen in the patientso with inoperable tumours, and rather less in the patients who underwent pneumonectomy. Bron $\Omega$ chial obstruction played a smaller part as perfu 
sion of the affected lobe was moderately or greatly reduced in 11 of 14 patients with a narrow or obstructed bronchus, but in 10 of these there was also a reduction in perfusion of the abjacent unobstructed lobe. Six other patients with normal bronchoscopies had large defects in perfusion indicating that bronchial obstruction is not the major cause of the reduced perfusion.

\section{DISCUSSION}

In patients with carcinoma of the bronchus, lung scanning provides an indirect assessment of the extent of involvement of the hilar regions by tumour tissue. As the tumour approaches the hilum, the relative perfusion of the affected lung decreases. The size of the defect in perfusion is more closely related to the amount of distortion or compression or invasion of the pulmonary vessels than to bronchial obstruction. Bronchial carcinomas derive most of their blood supply from the bronchial circulation, so that peripheral tumours show up as defects in the pattern of perfusion corresponding to their size on the chest radiograph. Small peripheral tumours, that is tumours less than $2-3 \mathrm{~cm}$ in diameter on the chest radiograph, are unlikely to be detected by scanning, for the resolution of the scanning system does not enable defects of this size to be distinquished from the surrounding radioactivity. However, a centrally placed tumour of similar size and even tumours invisible on the chest radiograph or on bronchoscopy may produce readily visible and sometimes unexpectedly large defects in perfusion because of involvement of the hilar vessels.

Our figures indicate that the relative perfusion of the affected lung tends to diminish as the tumour approaches the main bronchus, as judged by both bronchoscopy and the chest radiograph. In addition, the presence of extrathoracic metastases is associated with a considerable reduction in the relative perfusion of the affected lung.

Lung scanning can be of considerable value in the management of patients with carcinoma of the bronchus for it gives information about the relative perfusion of each lung and the regional perfusion within each lung that is not obtainable from either radiography, routine spirometry, or blood gas analysis. The more complicated technique of differential bronchospirometry also gives information about the relative perfusion of each lung, and the good correlation that exists between these two methods (Chernick et al., 1965 ; Rogers et al., 1967) has led Garnett and his colleagues to abandon differential bronchospirometry in favour of lung scanning (Garnett et al., 1969).
The relative perfusion of the tumour-bearing lung in all the patients with bronchoscopically inoperable tumours was less than one third of the total, and it was also below this figure in 10 of the 16 patients whose tumours were found to be unresectable at thoracotomy. The only indication of extensive hilar involvement by the tumour in these patients was the unexpected reduction in perfusion of the affected lung, for on clinical, bronchoscopic, and radiological grounds they were considered operable.

In only one patient out of 22 with a relative perfusion of less than $33.3 \%$ was it possible to remove the tumour (11 were bronchoscopically inoperable and 10 unresectable at thoracotomy) so that the chances of being able to resect a tumour in these circumstances are less than $5 \%$.

About one-third of patients undergoing thoracotomy are found to have unresectable tumours (Belcher and Anderson, 1965 ; Price Thomas, 1960) because of either extensive spread of the disease or invasion of vital structures which had not been appreciated preoperatively. Thirty-five per cent of our patients were found to have unresectable tumours, but this figure could have been reduced to $16.5 \%$ if thoracotomy had not been undertaken when the relative perfusion was less than $33.3 \%$. If pneumonectomy is contemplated the function of the remaining lung is clearly of vital importance. Abnormalities in perfusion of the unaffected lung are common and occurred in more than half of these patients. The majority of these defects $(68 \%)$ were relatively small but in 17 patients $(32 \%)$ they were larger. Sometimes such defects are large enough to contraindicate surgery (Garnett et al., 1968) or limit this to segmental resection when the tumour-bearing lung has the greater blood flow. In four of the patients who underwent thoracotomy, the defect in the pattern of perfusion in the unaffected lung was scored as larger than $25 \%$, but neither the chest radiograph nor spirometry gave any indication that such large defects would be found.

When lung scans are combined with the clinical picture, chest radiographs, bronchoscopy findings, and spirometry tests, the selection of patients for thoracotomy can be improved for, in addition to predicting when a tumour is likely to be inoperable and giving some indication as to whether a pneumonectomy or a lobectomy is the more probable, they may show unsuspected defects in the contralateral lung.

Lung scans, using radioactive particles, show the distribution of pulmonary arterial blood flow and because so many cardiorespiratory conditions dis- 
turb this flow a lung scan by itself is of little diagnostic value. Large and persistent defects in perfusion, in association with either peripheral or hilar shadows on the chest radiograph, make the diagnosis of malignant disease likely, but they do not establish it. Sometimes a lung scan will indicate the likely site of a carcinoma in that small group of patients $(5 \%$ of this series) with malignant cells in the sputum and normal chest radiographs and bronchoscopies. A single persistent defect in these circumstances should lead to further measures, such as angiography of the great veins and pulmonary arteries, in the hope of establishing the site more precisely.

We are grateful to Miss D. Nightingale, Professor R. S. Pilcher, Dr. H. Nicholson, Dr. P. J. D. Heaf, and Dr. P. D. B. Davies for allowing us to study patients under their care.

The photographs were kindly prepared by the University College Hospital Medical School Photographic Department.

This work was supported by a generous grant for technical assistance from the British Empire Cancer Campaign.

\section{REFERENCES}

Belcher, J. R., and Anderson, R. (1965). Surgical treatmen of carcinoma of the bronchus. Brit. med. J., 1, 948.
Chernick, V., Lopez-Majano, V., Wagner, H. N., Jr., and Dutton, R. E. Jr. (1965). Estimation of differential pulmonary blood flow by bronchospirometry and radioisotope scanning during rest and exercise. Amer. Rev. resp. Dis., 92, 958.

Garnett, E. S., Goddard, B. A., Fraser, H. S., and Macleod, W. M. (1968). Lung perfusion patterns in carcinoma of bronchus. Brit. med. J., 2, 209.

- Machell, E. S., and Macleod, W. M. (1969) Quantitated scintillation scanning for the measurement of lung perfusion. Thorax, 24, 372.

Hatch, H. B., Maxfield, W. S., and Ochsner, J. L. (1965). Radioisotope lung scanning in bronchogenic carcinoma. J. thorac. cardiovasc. Surg., 50, 634.

Price Thomas, C. (1960). Lobectomy with sleeve resection. Thorax, 15, 9.

Rogers, R. M., Kuhl, D. E., Hyde, R. W., and Mayock, R. L. 음 (1967). Measurement of the vital capacity and perfusion of each lung by fluoroscopy and macroaggregated albu- $c$ min lung scanning. Ann. intern. Med., 67, 947.

Sabiston, D. C. Jr., and Wagner, H. N. Jr. (1964). The diagnosis of pulmonary embolism by radioisotope scanning. Ann. Surg., 160, 575.

Secker Walker, R. H., Jackson, J. A., and Goodwin, J. (1970). Resolution of pulmonary embolism. Brit. med.J, , . 4, 135.

- and Provan, J. L. (1969). Scintillation scanning of lungs in preoperative assessment of carcinoma of bronchus. Brit. med. J., 3, 327.

Tauxe, W. N., Burchell, H. B., and Black, L. F. (1967). 응 Clinical applications of lung scanning. Mayo Clin. $\varrho$ Proc., 42, 473. 